\title{
Polymorphism of $N$-stearoylethanolamine: differential scanning calorimetric, vibrational spectroscopic (FTIR), and crystallographic studies
}

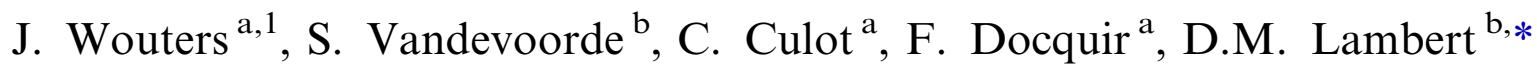 \\ ${ }^{a}$ Faculté des Sciences, Facultés universitaires Notre Dame de la Paix, Rue de Bruxelles, 61, 5000 Namur, Belgium \\ ${ }^{\mathrm{b}}$ Laboratoire de Chimie pharmaceutique et de Radiopharmacie, Ecole de Pharmacie, Faculté de Médecine, Université catholique de \\ Louvain, Avenue E. Mounier 73, UCL-CMFA 7340, B-1200 Brussels, Belgium
}

Received 17 September 2001; received in revised form 14 March 2002; accepted 12 April 2002

\begin{abstract}
Based on a series of physicochemical properties (differential scanning calorimetry, powder X-ray crystallographic studies and Fourier-transform infra red spectroscopic analysis) determined for $N$-stearoylethanolamine (NSEA) (C18:0) at different temperatures, evidence has been given that this compound can exist in (at least) three polymorphic forms. Powder X-ray crystallography clearly demonstrates the presence of three distinct molecular packings at distinct temperatures while spectral changes in the vibrational spectra reveal that the geometry of the $\mathrm{CH}_{2}-\mathrm{CO}$ functional group of the molecule is affected during the polymorphic transitions. Rationalization of the thermal physicochemical behavior of NSEA in terms of molecular packing is also proposed. It supposes rearrangement of the hydrocarbon chains upon heating of the molecule. (C) 2002 Elsevier Science Ireland Ltd. All rights reserved.
\end{abstract}

Keywords: Polymorphism; $N$-acylethanolamines; Differential scanning calorimetry; Vibrational spectroscopy; Powder diffraction

\section{Introduction}

Lipids and related organic long-chain compounds such as $n$-alkanes, $n$-alcohols, and fatty acids and their derivatives, appear in a wide

\footnotetext{
* Corresponding author. Tel.: +32-2-764-73-47; fax: +32-2764-73-63

E-mail address: lambert@cmfa.ucl.ac.be (D.M. Lambert).

${ }^{1}$ Present address: Institut de Recherches Microbiologiques Wiame - Av E Gryzon 1, 1070 Brussels, Belgium.
}

variety of structures depending on environmental conditions as well as on the thermal histories they have passed through. Such a complexity in structure is closely related to their functional activities and physicochemical properties (Hafez and Cullis, 2001). To know the molecular-level structures and the molecular mechanisms that control the stability of particular structures and phase changes is of fundamental importance in understanding the physicochemical behavior of such complicated systems. 
Among the lipids of interest in molecular pharmacology and physiology, $N$-acylethanolamines (NAES) are a class of lipid derivatives obtained from the condensation between fatty acids and ethanolamine. Terms of this family with a fatty acid longer than sixteen carbon atoms are known in mammals since some decades but their interest in pharmacology increases with the discovery of the C20:4 unsaturated homologous, $N$-arachidonoylethanolamine (C20:4), better known as anandamide (Devane et al., 1992). Anandamide is one of the endogenous ligands of the cannabinoid receptors (for a review Di Marzo et al., 1999) and it was recently proposed that it may act as endogenous ligand of the ligand-gated calcium channel, the vanilloid receptor VR1 (Zygmunt et al., 1999, 2000). Both unsaturated and saturated fatty acid ethanolamides probably share common biosynthetic pathways (for a review, Hansen et al., 2000).

$N$-stearoylethanolamine (NSEA, C18:0, Fig. 1) has been selected in this study. This molecule is produced in mammals, significant amounts have been detected in several tissues (Maccarrone et al., 2001). Little is known about the pharmacology of NSEA in mammals (Watanabe et al., 1999; Bueb et al., 2001) as most of the studies focused the three major species of NAEs, i.e. $N$-palmitoylethanolamine, $N$-oleoylethanolamine and anandamide (Hansen et al., 2000).

NSEA does neither bind to $\mathrm{CB}_{1}$ nor to $\mathrm{CB}_{2}$ cannabinoid receptors (Lambert and Di Marzo, 1999; Lambert et al., 1999) but is recognized by the fatty acid amide hydrolase (Jonsson et al., 2001). In our biological experiments, the solubility of this saturated fatty acid amide can vary from preparation to preparation. Therefore, the aim of our study was to investigate the possible existence of polymorphism of NSEA, by differential scanning calorimetry (DSC), powder X-ray crystallographic studies and Fourier-transform infra red (FTIR)

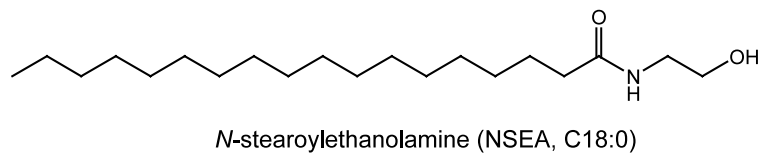

Fig. 1. Chemical diagram of NSEA. spectroscopic analysis. A putative rationalization of the thermal physicochemical behavior of that compound in terms of molecular packing is proposed.

\section{Experimental procedures}

\subsection{Synthesis of $N$-stearoylethanolamine}

NSEA was synthesized in one step by the addition of stearoyl chloride to a solution of freshly distilled ethanolamine in excess in dichloromethane at $4{ }^{\circ} \mathrm{C}$. The reaction mixture was stirred at room temperature and finally washed with water, $10 \%$ citric acid solution and brine. The organic layer was collected and dried over magnesium sulfate. After filtration, the solvent was removed under reduced pressure and the resulting powder was recrystallized in ethanol-water.

Melting points were determined on a Büchi SMP 20 capillary apparatus and were uncorrected. The purity of the compound was first checked by running TLC on aluminum-backed sheets of silica gel Merck $60 \mathrm{~F}_{254}$ using $\mathrm{KMnO}_{4}$ visualization.

Elemental analyses were performed on a Carlo Erba EA 1108 Analyzer (Carlo-Erba, Milano, Italy) and are within $\pm 0.4 \%$ of the theoretical values. ${ }^{13} \mathrm{C}$ NMR were recorded at ambient temperature on a AC-300 Bruker spectrometer. The chemical shifts are reported as $\delta(\mathrm{ppm})$ values relative to tetramethylsilane. Mass spectra were recorded on a Varian MAT $44 \mathrm{~S}$ (E.I. $70 \mathrm{eV}$, source $200{ }^{\circ} \mathrm{C}$ ), with the grateful help of Professor G.K.E. Scriba (Iena University, Germany).

NSEA m.p. $100-104{ }^{\circ} \mathrm{C}$, MS $\left[\mathrm{M}^{+}\right] 327,{ }^{13} \mathrm{C}-$ NMR $\left(\mathrm{CDCl}_{3}\right) 14.33\left(\mathrm{CH}_{3}\right), 23.42,26.67,30.06$, $30.14,30.30,30.44,32.73,37.00,42.68,61.56$ $\left(\mathrm{CH}_{2}\right), 176.35(\mathrm{C}=\mathrm{O})$, El. Analysis $\mathrm{C}, \mathrm{H}, \mathrm{N}$ for $\mathrm{C}_{20} \mathrm{H}_{41} \mathrm{NO}_{2}$.

\subsection{Differential scanning calorimetry}

Differential scanning calorimetric (DSC) studies were performed on a Perkin-Elmer DSC-4 differential scanning calorimeter equipped with a data station. The calorimeter was calibrated with a sample of indium $(99.99 \%$ pure, melting point 
$156.6{ }^{\circ} \mathrm{C}$ ). Aluminum pans were used for measurements. 1-3 mg of NSEA were weighted into the sample pan, covered with an aluminum lid and sealed by crimping. Reference pans were prepared without any sample in them. Heating and cooling scans were made from room temperature to $120{ }^{\circ} \mathrm{C}$ at different scan rates.

\subsection{Powder X-ray crystallographic studies}

For the X-ray powder diffraction measurements (PXRD), a Philips PW 1050 diffractometer (K $\alpha$ $\mathrm{Cu}=1.54178 \AA ; 2 \theta$ precision $=0.05^{\circ}$ ) equipped with a temperature control system (Philips PM 2522 A digital VA $\Omega$ meter and Pt probe; Philips, Eindhoven, The Netherlands) was used. The heating $\left(>20{ }^{\circ} \mathrm{C}\right)$ was performed with a TTK Anton Paar system (Anton Paar, Graz, Austria). The aluminum sample holder contained about $100 \mathrm{mg}$ of material. The thermal conditioning was determined on the basis of the DSC measurements. Detector scan speed was fixed to $1^{\circ} 2 \theta / \mathrm{min}$.

\subsection{Fourier-transform infra red spectroscopic analysis}

Infra red measurements were realized on a selfsupported sample wafer prepared by mixing $3 \mathrm{mg}$ of NSEA with $150 \mathrm{mg}$ of potassium bromide and pressing with a loading of 5 tons $/ \mathrm{cm}^{2}$. The sample wafer locked in a holder was placed in the infra red cell, which is made of Pyrex with two $\mathrm{CaF}_{2}$ windows and equipped with a system of oil circulation. A temperature control circuit had also been installed. The spectra were recorded using a Perkin-Elmer Fourier Transform Spectrum 2000 spectrometer. The sample was maintained at the desired temperature for $15 \mathrm{~min}$ for allowing it to reach an equilibrium before recording the spectra.

\subsection{Packing analysis and molecular modeling}

Coordinates of the crystal structure of N(2hydroxyethyl)octadecanamide, i.e. NSEA, were retrieved from the Cambridge Structure Databank (Allen and Kennard, 1993): reference code HXEODA. Molecular packing and intermolecular interactions analysis, vibrational spectroscopic spectra and powder diffraction patterns were performed with the Cerius suite of programs (MSI San Diego) on an Octane2 Silicon Graphics Workstation running Irix 6.5.

\section{Results}

\subsection{Differential scanning calorimetry}

Differential scanning calorigrams for the heating scans of solid-fluid phase transitions of NSEA are illustrated in Fig. 2.

In a single first heating scan, three peaks are observed in the differential scanning calorigrams (Fig. 2, top). The third peak coincides to the melting point of the compound and therefore this transition corresponds to the solid-liquid phase transition. This transition occurs at $102.5{ }^{\circ} \mathrm{C}$ for NSEA and confirms the melting point determined on a Büchi SMP 20 capillary apparatus. The observed melting point (III-Liq transition) is in agreement with earlier reports from the literature (Lambert et al., 1999; Ramakrishnan et al., 1997). The two other transitions which occur at temperatures below the main melting transitions (T I-II at $83{ }^{\circ} \mathrm{C}$ and $\mathrm{T}$ II-III at $93{ }^{\circ} \mathrm{C}$ ) correspond to transitions in the solid phase and allow the identification of (at least) three distinct polymorphic forms (termed I, II, and III) for NSAE. Ramakrishnan (Ramakrishnan et al., 1997) al-

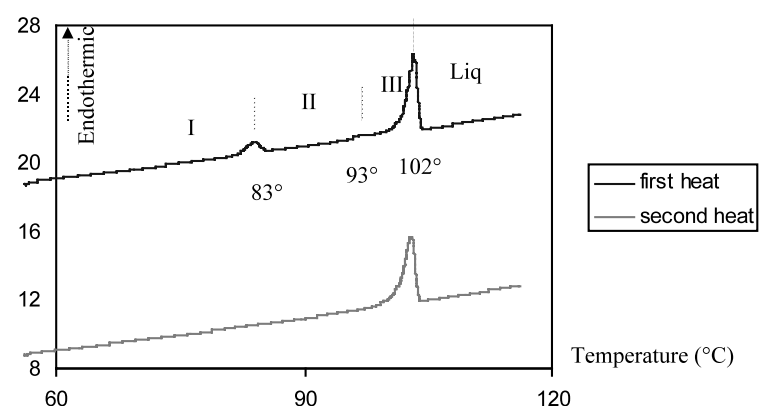

Fig. 2. Differential scanning calorigrams for the heating scans of solid-fluid phase transitions of NSEA. First heating (top) and second heating (bottom) scans are represented as a function of the temperature $\left({ }^{\circ} \mathrm{C}\right)$. Positive peaks correspond to endothermic transitions. 
ready noticed that all NAEs with acyl chains of 14-20 carbon atoms display two smaller transitions before the main melting transition in the first heating scan while $N$-decanoylethanolamine (C10:0) and $N$-lauroylethanolamine (C12:0) display only one additional transition that is rather broad. Our data are in complete agreement with this earlier report.

When the samples are subjected to further cycles of heating (and cooling) after the first heating and cooling scan, NSEA gives only a single sharp transition corresponding to the melting of the sample (III-Liq transition). This is contrary to what has been proposed by Ramakrishnan et al. Indeed this group reported that NEAs having acyl chain lengths of 16-20 C-atoms still present a second transition before the main melting transition. However, in their study, NEAs containing shorter acyl chains (8-14 C-atoms) gave only a single sharp transition, as we also observe for NSEA (C18:0).

Different scanning rates $\left(1-2.5{ }^{\circ} \mathrm{C} / \mathrm{min}\right)$ were tested in our study in the hope to detect any other transition before the melting point. But in all calorigrams, only one single sharp transition occurs after the first heating/cooling scan. No clear explanation is available. It is however not excluded that some sample impurities were present in the samples studied by Ramakrishnan et al. leading to the additional transition. Indeed, in the case of saturated monoacid triglycerides for example, it is known that as little as $2-3 \%$ impurities can markedly affect polymorphism, by stabilizing some forms in complex mixtures (Hoerr, 1964).

\subsection{Powder X-ray crystallographic studies}

In order to further approach structural aspects of the polymorphism of NSEA, powder X-ray diffractograms were recorded at different temperatures, corresponding to the I $\left(80{ }^{\circ} \mathrm{C}\right)$, II $(85$ and $\left.90{ }^{\circ} \mathrm{C}\right)$, or III $\left(95{ }^{\circ} \mathrm{C}\right)$ polymorphic forms. Those diffractograms are compared in Fig. 3.

The diffractograms collected at 80,85 and $95{ }^{\circ} \mathrm{C}$ present different patterns of diffraction in the short spacing region $\left(16<2 \theta<26^{\circ}\right)$ of the spectra. This region corresponds to short reticular distances between hydrocarbon chains and/or polar groups. As will be discussed later in the text, those distances depend on the subcell and are directly related to the arrangement of the molecules in the solid state. The differences in the diffractograms clearly demonstrate the presence of

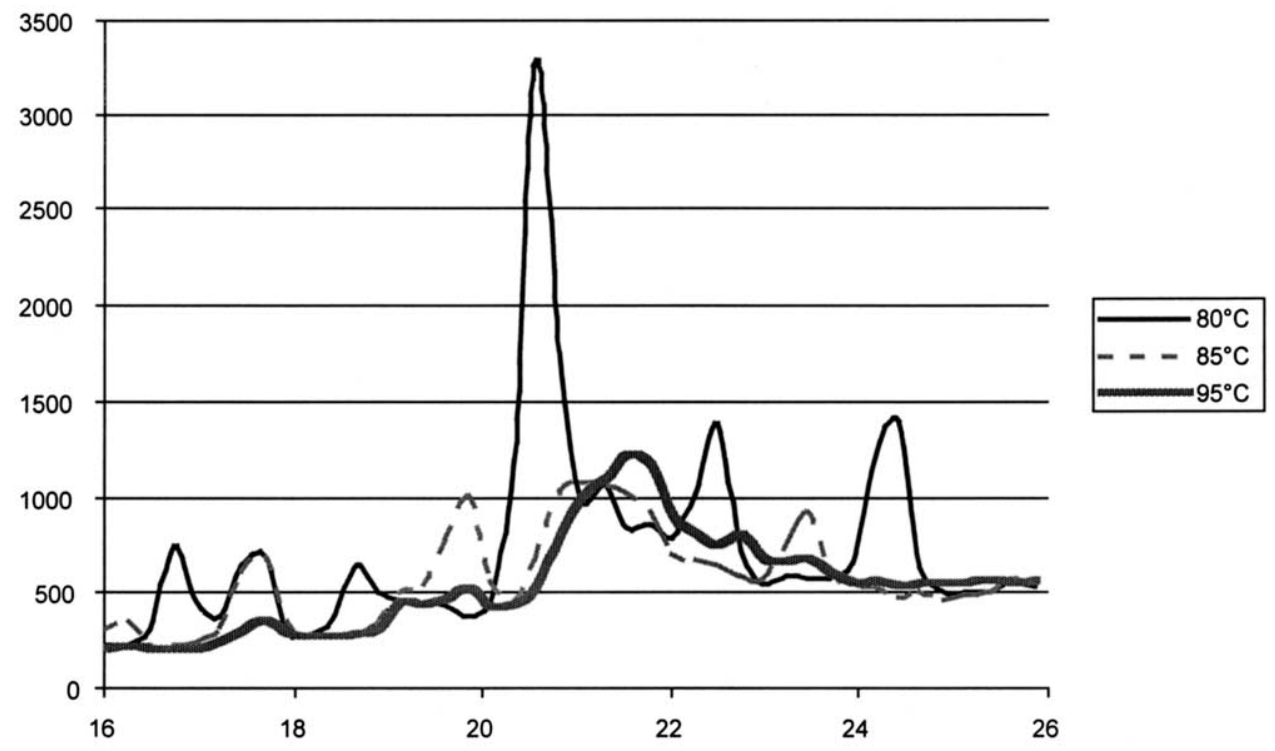

Fig. 3. X-ray diffraction profile of NSEA in the range of $16<2 \theta<26^{\circ}$ (short spacing region of the spectra) at different temperatures. 
Table 1

X-ray powder diffraction values characteristic of NSEA for different temperatures

\begin{tabular}{lll}
\hline Temperature & $d(\AA)$ & $2 \theta\left({ }^{\circ}\right)$ \\
\hline $80 \quad{ }^{\circ} \mathrm{C}$ & $5.3(\mathrm{w})^{\mathrm{a}}$ & 16.7 \\
$($ Form I) & $5.1(\mathrm{w})$ & 17.5 \\
& $4.3(\mathrm{~s})$ & 20.5 \\
& $3.9(\mathrm{~m})$ & 22.4 \\
& $3.6(\mathrm{~m})$ & 24.3 \\
$85 \quad{ }^{\circ} \mathrm{C}=90 \quad{ }^{\circ} \mathrm{C}$ & $5.1(\mathrm{w})$ & 17.5 \\
$($ Form II) & $4.5(\mathrm{~m})$ & 19.7 \\
& $4.2(\mathrm{~m})$ & 20.9 \\
& $3.8(\mathrm{w})$ & 23.3 \\
$95{ }^{\circ} \mathrm{C}$ & $(5.1(\mathrm{vw}))$ & 17.5 \\
$($ Form III) & $4.1(\mathrm{~m})$ & 21.5 \\
\hline
\end{tabular}

a s, strong; m, medium; w, weak; vw, very weak.

three distinct molecular packings, existing at given temperatures.

The following high-intensity diffraction peaks are characteristic to each polymorphic form at: $2 \theta=16.7^{\circ}, 17.5^{\circ}, 20.5^{\circ}, 22.4^{\circ}, 24.3^{\circ}$ for Form I; $2 \theta=17.5^{\circ}, 19.7^{\circ}, 20.9^{\circ}, 23.3^{\circ}$ for Form II; $2 \theta=$ $17.5^{\circ}$ for Form III. The corresponding reticular distances are given in Table 1.

Spectra were also recorded in the long spacing region $\left(2 \theta\right.$ values under $\left.10^{\circ}\right)$ at 80,85 , and $95{ }^{\circ} \mathrm{C}$. For Form I $\left(80{ }^{\circ} \mathrm{C}\right)$ diffraction peaks are observed for $2 \theta$ values of $40.4^{\circ}(n=1)$ and $9.3^{\circ}(n=4)$ corresponding to a value of long spacing of about $39 \AA$ A. For Form II $\left(85^{\circ} \mathrm{C}\right)$ peaks are present at $2 \theta=41.6^{\circ}(n=1)$ and $15.6^{\circ}(n=3)$ corresponding to a longer value of the long spacing (about $44 \AA$ ) for this form. No defined diffraction pattern is observed at $95{ }^{\circ} \mathrm{C}$.

\subsection{Fourier-transform infra red spectroscopic analysis}

Along with X-ray and neutron diffraction methods, vibrational spectroscopy is a powerful tool for the investigation of polymorphism (Kabayashi, 1988). In the case of fatty acids and related compounds having a polar head group and a chain end, the infra red (IR) series due to methylene wagging modes (1150-1400 $\mathrm{cm}^{-1}$ ) and the methylene rocking-twisting modes (700$1050 \mathrm{~cm}^{-1}$ ) are the most commonly used. In particular, the appearance of the well-defined band series indicates the all-trans conformation of the alkyl chains.

The FTIR spectra of NSEA between 4000 and $600 \mathrm{~cm}^{-1}$ have been recorded at different temperature: $50,75,80,85,90,95$, and $102{ }^{\circ} \mathrm{C}$. A partial view of those spectra in the 1500-1200 $\mathrm{cm}^{-1}$ region is given in Fig. 4.

The $1500-1200 \mathrm{~cm}^{-1}$ region, corresponding to $\mathrm{CH}_{2}-\mathrm{CO}$ vibrations, presents the most marked differences. In particular, a band around 1460 $\mathrm{cm}^{-1}$ appears as a doublet below $80{ }^{\circ} \mathrm{C}$ and becomes a singlet above that temperature. This is again related to the conformational changes that occur at the molecular level and gives rise to distinct polymorphic forms. The spectra of Form

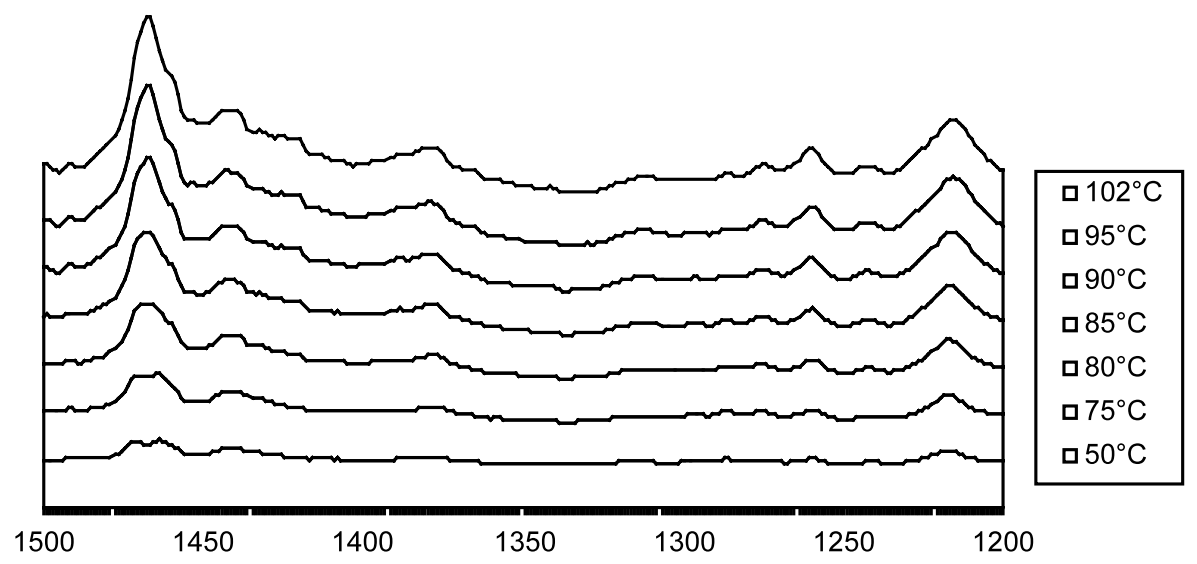

Fig. 4. IR absorption spectra of NSEA recorded at different temperatures in the $1500-1200 \mathrm{~cm}^{-1}$ region. 
II (spectra at 85 and $90{ }^{\circ} \mathrm{C}$ ) more closely resembles the one of Form III than of Form I. This means that the conformational change is more important for the I-II transition than for the IIIII transition, as already suggested by the crystallographic study.

\subsection{Molecular packing analysis of NSEA}

The coordinates of the crystal structure of NSEA obtained by single crystal X-ray diffraction (Dahlen et al., 1977) are available from the Cambridge Structure DataBase (Refcode HXEODA). $N$-(2-hydroxyethyl)octadecanamide (NSEA) crystallized in the Pc space group with unit cell dimensions: $a=47.588, b=4.886, c=9.005 \AA$, $\beta=93.86^{\circ}$. There are two molecules in the asymmetric unit leading to four molecules in the monoclinic unit cell.

The hydrocarbon chain-packing of NSEA belongs to an orthorhombic perpendicular $(\mathrm{O} \perp)$ chain packing, meaning that the zig-zag planes of the different chains are perpendicular $(\perp)$ and arranged in an orthorhombic subcell. The molecules are organized in a tail-to-tail fashion, similar to the arrangement expected in a bilayer membrane. A similar arrangement is also found in the structure of $N$-myristoylethanolamine (Ramakrishnan and Swamy, 1999). The hydrophobic saturated hydrocarbon chain of NSEA chains adopts an all-trans zig-zag conformation in the crystalline phase, except for the $\mathrm{C}-\mathrm{C}$ bonds very close to the polar group. In particular, torsion angle $\mathrm{C} 3-\mathrm{C} 4-\mathrm{C} 5-\mathrm{C} 6$ is 71.14 and $-67.71^{\circ}$ for the two molecules in the asymmetric unit respectively. As a consequence, the hydrophobic chains are tilted with respect to the bilayer as illustrated in Fig. 5. The methyl ends of the stacked bilayers are in van der Waals contacts.

In addition to the van der Waals interactions, each hydroxyl group forms two hydrogen bonds with the $\mathrm{OH}$ groups of related molecules. These $\mathrm{OH}$. . .O hydrogen bonds form an extended zig-zag type network in the crystal packing. There are also strong hydrogen bonds between the amide $\mathrm{N}-\mathrm{H}$ and carbonyl oxygen of adjacent NSEA molecules.

The short spacing values measured at $80{ }^{\circ} \mathrm{C}$ (Fig. 3, Table 1) correspond to geometries ob-

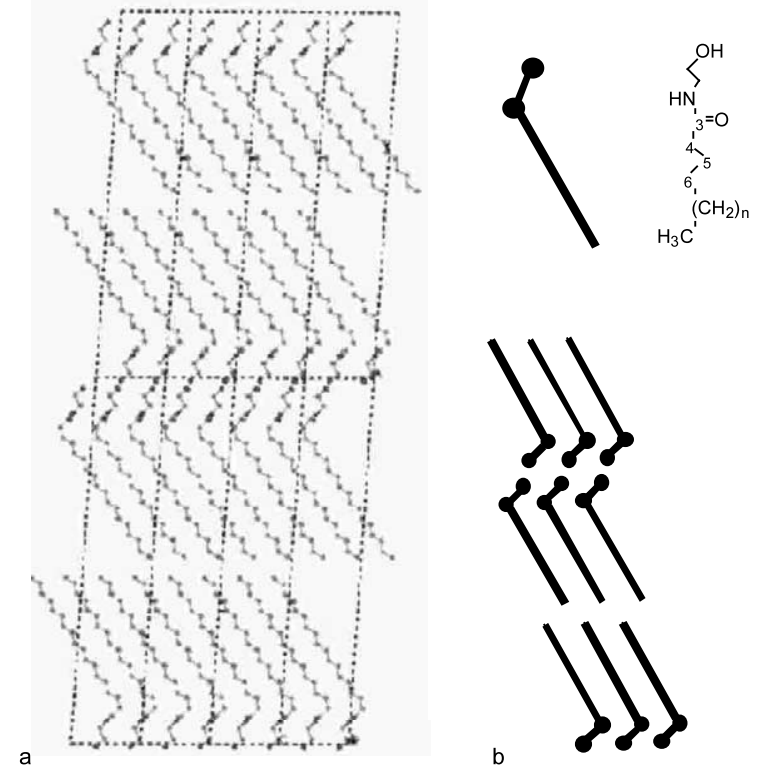

Fig. 5. Packing diagrams of NSEA viewed in the $a c$ plan (a) and schematic representation of the molecular packing (b).

served in the crystallographic structure reported for NSEA. Indeed the closest methyl-methyl contact distances between the opposing layers is $4.2 \AA$. The distance between the methyl groups of adjacent molecules in the same layers is about 5.8 $\AA$. This is consistent with classical short spacing values characterizing orthorhombic chain packing subcells (Hernqvist, 1988).

In the polar heads, strongly connected by a network of $\mathrm{H}$ bonds, the carbonyl $\mathrm{O}$ binds the amide $\mathrm{N}(2.81 \AA)$ while the distance between the hydroxyl groups is about $2.7 \AA$. This suggests that the molecular packing deduced from the single crystal structure corresponds to the Form I polymorphic structure. This is reasonable, as it is the form that is stable at room temperature and that will first crystallize.

\section{Discussion}

\subsection{Experimental evidence of the existence of polymorphic structures}

On the basis of the calorimetric (i.e. chain melting) properties of NSEA it can be observed 
that additional endothermic transitions (transitions I-II and II-III, Fig. 2) take place at temperatures below the main transition (III- Liq transition at $102{ }^{\circ} \mathrm{C}$, Fig. 2). These transitions must be related to polymorphic crystalline transition. Similar transitions are observed also for fatty acids and alkanes (Small, 1986; Marsh and Swamy, 2000). The transition temperatures therefore serve to delineate temperature ranges of stability for the distinct polymorphic forms: Form I below $83{ }^{\circ} \mathrm{C}$, Form II between 83 and $93{ }^{\circ} \mathrm{C}$, Form III between 93 and $102{ }^{\circ} \mathrm{C}$. Above $102{ }^{\circ} \mathrm{C}$ NSEA melts.

Powder X-ray diffraction patterns (Fig. 3) and IR vibrational spectra (Fig. 4) have been recorded for the three polymorphic forms. The spectra of Form II more closely resembles those of Form III than Form I. This means that the conformational change is more important for the I-II transition than for the II-III transition. Those conformational changes directly affect the arrangement of the molecules in the solid state (subcell) as reflected by the changes in the short spacing region $\left(16<2 \theta<26^{\circ}\right)$ of the diffractograms (Fig. 3). The polymorphic transition also deeply affects the vibrational spectra of NSEA, the 1500-1200 $\mathrm{cm}^{-1}$ region, corresponding to $\mathrm{CH}_{2}-\mathrm{CO}$ vibrations, presenting the most marked differences (Fig. 4). The IR spectral changes induced by the heating can be interpreted as follows; doublet to single bands correspond respectively to vibrational modes in either two distinct or only one plane. In Form I $\left(<83{ }^{\circ} \mathrm{C}\right)$, the vibration of the $\mathrm{CH}_{2}-$ $\mathrm{CO}$ occurs in two distinct planes, suggesting two possible arrangements for this form in the crystal packing. In Form III (spectra at $95{ }^{\circ} \mathrm{C}$ ), the vibration at $1460 \mathrm{~cm}^{-1}$ gives rise to a single band, suggesting that the vibrational mode of the $\mathrm{CH}_{2}-\mathrm{CO}$ function group lies in only one plane.

\subsection{Possible mechanism of polymorphic transitions}

Rationalization of the conformational changes that affect the molecules and their crystal packing upon heating is proposed in terms of molecular packing analysis of the crystal structure of NSEA. Those changes can explain most of the experi- mental data obtained for the different polymorphic forms.

The polymorphic form (I), stable at room temperature, is the one that is observed in the single crystal structure of NSEA. Short spacing values measured at $80{ }^{\circ} \mathrm{C}$ (Fig. 3 and Table 1) correspond to interatomic distances in the crystallographic structure reported for NSEA.

The existence of alternative crystal structures is a characteristic property of long chains compounds (fatty acids, triglycerides,...). This is due to the possibilities for different chain packing arrangements, and furthermore, for variations in the tilt of the chains within the layers. In the particular case of triglycerides, polymorphism is associated to crystal packing changes from a perpendicular subcell to a parallel one upon heating (Culot, 1999). Heating thus allows rearrangement of the hydrocarbon chains. A similar behavior is proposed in the case of NSEA and is illustrated in Fig. 6. The geometry of the polar heads of the molecules would not be very much affected in the beginning of the heating process as they are held by a network of $\mathrm{H}$ bondings. This is reflected by the presence of fixed short spacing values common to all three I, II and II polymorphic forms in the PXRD patterns. From our structural interpretation, changes in tilt angles of the hydrocarbon chains relative to the layer plane must lead to changes in the small-angle region of the PXRD patterns (long spacing). Indeed, differences in values of long spacings ( 39 and $44 \AA$ at 80 and $85{ }^{\circ} \mathrm{C}$ respectively) are observed between
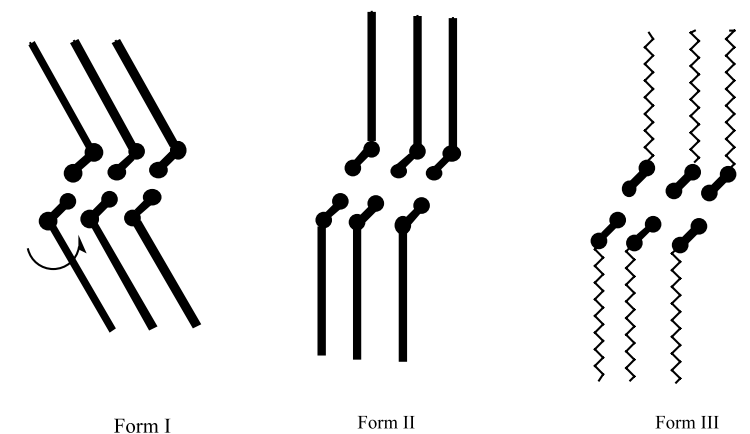

Fig. 6. Hypothetical molecular arrangements associated to the different polymorphic forms observed for NSEA. 
Forms I and II and they confirm a different longitudinal packing for those two forms.

Most of the rearrangement would affect the $\mathrm{CH}_{2}-\mathrm{CO}$ function following rotation of the acyl chain upon heating. This suggestion is in good agreement with the FTIR data. Indeed, the 1500$1200 \mathrm{~cm}^{-1}$ region corresponding to $\mathrm{CH}_{2}-\mathrm{CO}$ vibrations presents the most marked differences. The deeper rearrangement of the hydrocarbon chains should be associated to the Form I-II transition as the vibrational band around 1460 $\mathrm{cm}^{-1}$ appears as a doublet below $80{ }^{\circ} \mathrm{C}$ and becomes a singlet above that temperature. In Forms II and III, all lateral hydrocarbon chains are parallel in the crystal packing and vibrate in a single plane.

We further propose that Form II-III transition destabilized the molecular packing leading to a 'liquid crystalline' structure for Form III, where the lateral hydrocarbon chains are free to rotate. This could explain why the powder diffraction pattern (Fig. 3 and Table 1) exhibits less lines at $95{ }^{\circ} \mathrm{C}$. At that temperature short spacing values correspond to mean distances between free rotating hydrocarbon chains.

Form III thus approaches the liquid state that would be obtained after further heating above the melting point. During the whole hypothetical process, from Form I to the liquid state, entropy is increased.

\section{Conclusions}

Based on a series of physicochemical properties (DSC, powder X-ray crystallographic studies and FTIR spectroscopic analysis) determined for NSEA (C18:0) at different temperatures, evidence has been given that this compound can exist under (at least) three polymorphic forms.

On the basis of the calorimetric (i.e. chain melting) properties it can be concluded that additional endothermic transitions take place at temperatures below the main transition $\left(102{ }^{\circ} \mathrm{C}\right)$ and that these transitions must be related to polymorphic crystalline transition. The observed transition temperatures therefore serve to delineate temperature ranges of stability for the distinct polymorphic forms: Form I below $83{ }^{\circ} \mathrm{C}$, Form II between 83 and $93{ }^{\circ} \mathrm{C}$, Form III between 93 and $102{ }^{\circ} \mathrm{C}$.

The diffractograms collected at 80,85 and $95{ }^{\circ} \mathrm{C}$ on powder samples of NSEA are different and clearly demonstrate the presence of three distinct molecular packings at distinct temperatures.

The spectra of Form II more closely resembles the one of Form III than Form I suggesting that the conformational change is more important for the I-II transition than for the II-III transition.

Spectral changes in the vibrational spectra in the $1500-1200 \mathrm{~cm}^{-1}$ region, appear upon heating of NSEA. In particular, a band around $1460 \mathrm{~cm}^{-1}$ appears as a doublet below $80{ }^{\circ} \mathrm{C}$ and becomes a singlet above that temperature. This suggests that the geometry of the $\mathrm{CH}_{2}-\mathrm{CO}$ functional group of the molecule is affected during the polymorphic transitions.

A putative rationalization of the thermal physicochemical behavior of NSEA in terms of molecular packing has been proposed. It supposes rearrangement of the hydrocarbon chains upon heating of the molecule. This hypothesis is based on an in depth analysis of the crystal packing of the compound obtained by single crystal crystallography. It is in agreement with the experimental data obtained for NSEA and incorporates observations deduced for related long chains compounds (fatty acids, triglycerides,...).

\section{Acknowledgements}

Authors acknowledge Professor Bao Lian Su for access to FTIR spectroscopy facilities and expertise. Part of this work received financial support from the DGTRE (Region Wallonne) by First Spin off Project 001/4602.

\section{References}

Allen, F.H., Kennard, O., 1993. Systematic analysis of structural data as a research technique in organic chemistry. Acc. Chem. Res. 6, 146-153. 
Bueb, J.L., Lambert, D.M., Tshirhart, E.J., 2001. Receptorindependent effects of natural cannabinoids in rat peritoneal mast cells in vitro. Biochem. Biophys. Acta 1538, 252-259.

Culot, C., 1999. Molecular modeling of triglycerides polymorphism. Recent Res. Devel. Oil Chem. 3, 107-139.

Dahlen, B., Pascher, I., Sundell, S., 1977. The crystal structure of $N$ (2-hydroxyethyl)octadecanamide. Acta Chem. Scand. Ser. A 31, 313-320.

Devane, W.A., Hanus, L., Breuer, A., Pertwee, R.G., Stevenson, L.A., Griffin, G., Gibson, D., Mandelbaum, A., Etinger, A., Mechoulam, R., 1992. Isolation and structure of a brain constituent that binds to the cannabinoid receptor. Science 258, 1946-1949.

Di Marzo, V., Bisogno, T., De Petrocellis, L., Melck, D., Martin, B.R., 1999. Cannabimimetic fatty acid derivatives: the anandamide family and other endocannabinoids. Curr. Med. Chem. 6, 721-744.

Hafez, I.M., Cullis, P.R., 2001. Roles of lipid polymorphism in intracellular delivery. Adv. Drug Delivery Rev. 47, 139148.

Hansen, H.S., Moesgaard, B., Hansen, H.H., Petersen, G., 2000. $N$-acylethanolamines and precursor phospholipidsrelation to cell injury. Chem. Phys. Lipids 108, 135-150.

Hernqvist, L., 1988. Crystal structures of fats and fatty acids. In: Garti, N., Sato, K. (Eds.), Crystallization and Polymorphism of Fats and Fatty Acids. Marcel Dekker, Inc, New York, pp. 97-137.

Hoerr, C.W., 1964. X-ray diffraction of fats. J. Am. Oil Chem. Soc. 41 (Suppl. 7), 4.

Jonsson, K.O., Vandevoorde, S., Lambert, D.M., Tiger, G., Fowler, C.F., 2001. Effects of homologues and analogues of palmitoylethanolamide upon the inactivation of the endocannabinoid anandamide. Br. J. Pharmacol. 133, $1263-$ 1275.

Kabayashi, M., 1988. Vibrational spectroscopic aspects of polymorphism and phase transitions of fats and fatty acids. In: Garti, N., Sato, K. (Eds.), Crystallization and Polymorphism of Fats and Fatty Acids. Marcel Dekker, Inc, New York, pp. 139-187.

Lambert, D.M., Di Marzo, V., 1999. The palmitoylethanolamide and oleamide enigmas: are these two fatty acid amides cannabimimetic? Curr. Med. Chem. 6, 739-755.
Lambert, D.M., DiPaolo, F.G., Sonveaux, P., Kanyonyo, M.R., Govaerts, S.J., Hermans, E., Bueb, J.L., Delzenne, N.M., Tschirhart, E.J., 1999. Analogues and homologues of $N$-palmitoylethanolamide, a putative $\mathrm{CB} 2$ endogenous agonist: synthesis and binding potency to the rat spleen cannabinoid receptors. Biochem. Biophys. Acta 1440, $266-$ 274.

Maccarrone, M., Attina, M., Cartoni, A., Bari, M., FinazziAgro, A., 2001. Gas chromatography-mass spectrometry analysis of endogenous cannabinoids in healthy and tumoral human brain and human cells in culture. $\mathbf{J}$. Neurochem. 76, 594-601.

Marsh, D., Swamy, M.J., 2000. Derivatised lipids in membranes. Physicochemical aspects of $N$-biotinyl phosphatidylethanolamines, $N$-acyl phosphatidylethanolamines and $N$-acyl ethanolamines. Chem. Phys. Lipids 105, 43-69.

Ramakrishnan, M., Sheeba, V., Komath, S.S., Swamy, M.J., 1997. Differential scanning calorimetric studies on the thermotropic phase transitions of dry and hydrated forms of $N$-acylethanolamines of even chain lengths. Biochim. Biophys. Acta (Biomembranes) 1329, 302-310 (Corrigendum appears in 1998 Biochim. Biophys. Acta (Biomembranes) 1371, 382).

Ramakrishnan, M., Swamy, M.J., 1999. Molecular packing and intermolecular interactions in $N$-acylethanolamines: crystal structure of $N$-myristoylethanolamine. Biochim. Biophys. Acta 1418, 261-267.

Small, D.M., 1986. The physical chemistry of lipids: from alkanes to phospholipids. In: Small, D.M. (Ed.), Handbook of Lipid Research. Plenium Press, New York, pp. 245-392.

Watanabe, K., Matsunaga, T., Nakamura, S., Kimura, T., Ho, I.K., Yoshimura, H., Yamamoto, I., 1999. Pharmacological effects in mice of anandamide and its related fatty acid ethanolamides, and enhancement of cataleptogenic effect of anandamide by phenylmethylsulfonyl fluoride. Biol. Pharm. Bull. 22, 366-370.

Zygmunt, P.M., Petersson, J., Andersson, D.A., Chuang, H., Sørgård, M., Di Marzo, V., Julius, D., Högestätt, E.D., 1999. Vanilloid receptors on sensory nerves mediate the vasodilator action of anandamide. Nature 400, 452-457.

Zygmunt, P.M., Julius, D., Di Marzo, V., Högestätt, E.D., 2000. Anandamide - the other side of the coin. Trends Pharmacol. Sci. 212, 43-44. 